\title{
SPHERICAL STEIN MANIFOLDS AND THE WEYL INVOLUTION
}

\author{
Dmitri Akhiezer
}

\section{Introduction}

Let $G$ be a connected reductive algebraic group over $\mathbb{C}$. A normal algebraic $G$-variety $X$ is called spherical if a Borel subgroup $B \subset G$ has an open orbit in $X$. For $X$ affine this is the case if and only if the function algebra $\mathbb{C}[X]$ is a multiplicity free $G$-module, i.e., if and only if every irreducible $G$-module appears in $\mathbb{C}[X]$ at most once; see [Se], $[\mathrm{VK}]$.

The notion of a spherical variety has been carried over to the category of complex spaces. Namely, let $X$ be a normal complex space and $K$ a connected compact Lie group acting on $X$ by holomorphic transformations. Every element of the complexified Lie algebra $\mathfrak{g}=\mathfrak{k} \otimes \mathbb{C}$ gives rise to a holomorphic vector field on $X$. The complex $K$-space $X$ is called spherical if there exists a point $x \in X$ such that the holomorphic tangent space $T_{x}(X)$ is generated by the vector fields from a Borel subalgebra $\mathfrak{b} \subset \mathfrak{g}$. Similarly to the algebraic setting, a normal Stein $K$-space is spherical if and only if the Fréchet $K$-module $\mathcal{O}(X)$ is multiplicity free, i.e., every irreducible $K$-module either occurs in $\mathcal{O}(X)$ with multiplicity 1 or does not occur at all. Moreover, in that case $X$ is a $K$-invariant domain in a spherical affine $K^{\mathbb{C}}$-variety; see $[\mathrm{AH}]$.

In order to state our main results, we recall some terminology. Given a group $\Gamma$ acting on two sets $X, Y$ and an automorphism $\alpha$ of $\Gamma$, we say that a map $\mu: X \rightarrow Y$ is $\alpha$-equivariant if $\mu(\gamma x)=\alpha(\gamma) \mu(x)$ for all $x \in X, \gamma \in \Gamma$. A self-map $\mu: X \rightarrow X$ is called an involution if $\mu^{2}=\mathrm{Id}$. By an involution of a group we will always mean an involution which is a group automorphism.

In this paper we consider only non-singular Stein $K$-spaces. Our goal is a characterization of spherical $K$-manifolds in terms of $K$-equivariant antiholomorphic involutions.

The author was supported by the Russian Foundation for Basic Research, Grant 07-0100230, and by SFB/TR12 "Symmetrien und Universalität in mesoskopischen Systemen" of the Deutsche Forschungsgemeinschaft. 
Theorem 1.1 Let $X$ be a Stein manifold on which a connected compact Lie group $K$ acts by holomorphic transformations. Then $X$ is spherical if and only if there exists an antiholomorphic involution $\mu: X \rightarrow X$ such that

$$
\mu(x) \in K x
$$

for all $x \in X$.

According to a theorem of J.Faraut and E.G.F.Thomas, the existence of $\mu$ with the above properties is sufficient for $X$ to be spherical; see [FT], Theorem 3. In fact, the setting in $[\mathrm{FT}]$ is more general, namely, the manifold $X$ need not be Stein and the group $K$ is not necessarily compact. The conclusion is that, in the presence of $\mu$ satisfying (1), any irreducible unitary $K$-module occurs in $\mathcal{O}(X)$ with multiplicity $\leq 1$. A simplified proof in our context is found in $[\mathrm{AP}]$. The converse statement is known in one special case, namely, if $X$ is an affine variety homogeneous under the complexified group $G=K^{\mathbb{C}}$; see $[\mathrm{AP}]$.

We recall the definition of a Weyl involution in Sect.2. Let $\theta$ be a Weyl involution of $K$. The crucial role in our considerations is played by $\theta$-equivariant antiholomorphic involutions of Stein manifolds acted on by $K$.

Theorem 1.2 Let $K$ be a connected compact Lie group, $\theta: K \rightarrow K$ a Weyl involution, and $X$ a Stein manifold on which $K$ acts by holomorphic transformations. Assume that $X$ is spherical with respect to $K$. Then there exists a $\theta$-equivariant antiholomorphic involution $\mu: X \rightarrow X$.

In the paper of E.B.Vinberg and the author, the Weyl involution appears as a tool bringing the notion of weak symmetry into the theory of spherical varieties. If $X$ is homogeneous under $G=K^{\mathbb{C}}$ then Theorem 1.2 is a consequence of Theorem 5.11 in $[\mathrm{AV}]$. At this stage, the proof relies upon the classification of reductive spherical subgroups in simple groups given by Krämer's list. In the general case, $X$ embeds as a $K$-invariant domain into the space of a vector bundle $G \times_{H} V$, where $H$ is a reductive spherical subgroup in $G$ and $V$ is a spherical $H$-module (see Sect.5). Therefore we need an elementary version of Theorem 1.2 for a $K$-module $X=V$, which we will then apply to the fiber. As long as $K$ is connected, this result does not require sphericity of the $K^{\mathbb{C}}$-module $V$. Moreover, Theorem 3.1 shows that there exists a $\theta$-equivariant involution $\mu: V \rightarrow V$, which is in fact antilinear, and that such a $\mu$ is essentially unique if $V$ is irreducible. However, in order to accomplish the proof of Theorem 1.2, one must be able to apply Theorem 3.1 to possibly disconnected groups acting on the fiber $V$. Our argument is based on Theorem 4.3. Here is its simplified version, which might be of independent interest.

Let $G / H$ be a spherical homogeneous space, $\sigma: G \rightarrow G$ an involution defining a split real form of $G$, and $U$ a Lie subgroup of $G$ containing $H$, such that $H \triangleleft U$ and $U / H$ is compact. If $\sigma(H)=H$ then $\sigma(U)=U$ and the action of $\sigma$ on the (abelian) group $U / H$ is the inversion. 
It is interesting to compare this statement with Proposition 5.2 in [AV] (see also Corollary 4.4). The proof of main results is given in Sect.5.

Throughout the paper, we denote by $N_{G}(H)$ the normalizer of a subgroup $H$ in a group $G$, by $G^{\circ}$ the connected component of the neutral element $e$ in a topological group $G$, and by $\mathrm{X}(G)$ the character group of an algebraic group $G$.

\section{Preliminaries}

Let $G$ be a connected reductive algebraic group over $\mathbb{C}$. Recall that an involutive regular automorphism $\theta: G \rightarrow G$ is called a Weyl involution if there exists a maximal torus $T \subset G$ such that $\theta(t)=t^{-1}$ for $t \in T$. It is known that such an involution exists and that any two Weyl involutions of $G$ are conjugate by an inner automorphism; see e.g. [He], Ch. IX, $\S 5$. From the point of view of the representation theory, the main property of a Weyl involution is the following one. Let $V$ be a rational $G$-module, $\varrho: G \rightarrow \mathrm{GL}(V)$ the corresponding representation, and $V^{\theta}$ the $G$-module given by the representation $g \mapsto \varrho(\theta(g))$ in the same vector space $V$. Then $V^{\theta}$ is isomorphic to the dual $G$-module $V^{*}$.

Let $G^{\theta} \subset G$ be the fixed point subgroup of $\theta$. This is a symmetric algebraic subgroup of $G$, which is always reductive. One can find a Cartan involution $\tau$ commuting with $\theta$ or, equivalently, a maximal compact subgroup $K \subset G$ stable under $\theta$. Any two maximal compact subgroups with this property are conjugate by an element of $G^{\theta}$. Moreover, one can arrange that such a subgroup contains the maximal compact subgroup $T_{c} \subset T$; see [He], Ch. VI, A8 or Lemma 2.2 below. Thus, for any choice of $K$ satisfying $\theta(K)=K$ there is an element $a \in G^{\theta}$ such that $K$ contains the compact torus $a T_{c} a^{-1}$. Since $\sigma=\tau \theta$ preserves this torus and acts on it as the inversion, the fixed point subgroup of $\sigma$ is a split real form of $G$.

In what follows, we will keep the above notation. Namely, we will consider three involutive automorphisms of $G$. These are a Weyl involution $\theta$, a Cartan involution $\tau$ commuting with $\theta$ and the product $\sigma=\theta \tau=\tau \theta$. The fixed point subgroups $K=G^{\tau}$ and $G^{\sigma}$ are a compact real form and, respectively, a split real form of $G$. We remark that the notion of a Weyl involution can also be defined for connected compact Lie groups. The definition is similar and its meaning for the representation theory is similar as well. Note that the restriction $\left.\theta\right|_{K}$ is a Weyl involution of $K$.

For the convenience of the reader we recall the proof of two simple facts about Lie groups with finitely many connected components which we will need in the sequel.

Lemma 2.1 Let $\pi: G_{1} \rightarrow G_{2}$ be an epimorphism of Lie groups. If Ker $\pi$ has finitely many connected components and $K_{2} \subset G_{2}$ is a compact subgroup then there exists a compact subgroup $K_{1} \subset G_{1}$ such that $K_{2}=\pi\left(K_{1}\right)$.

Proof Without loss of generality assume that $G_{2}=K_{2}$. Then $G_{1}$ has finitely many connected components. We have $K_{2}^{\circ}=\pi\left(G_{1}^{\circ}\right)$. By a theorem of D.Montgomery there exists a compact subgroup $L \subset G_{1}^{\circ}$ such that $\pi(L)=K_{2}^{\circ}$; see $[\mathrm{M}]$, Cor. 2 . Let $K_{1}$ be a 
maximal compact subgroup of $G_{1}$ such that $L \subset K_{1}$. Then $G_{1}=K_{1} \cdot G_{1}^{\circ}$ by a theorem of G.D.Mostow; see [Mo]. Hence $K_{2}=\pi\left(G_{1}\right)=\pi\left(K_{1}\right) \cdot K_{2}^{\circ}=\pi\left(K_{1}\right)$.

Lemma 2.2 Let $G_{1} \subset G_{2}$ be two Lie groups with finitely many connected components, $G_{1}$ a closed subgroup of $G_{2}$, and $\Delta$ a finite group of automorphisms of $G_{2}$, which leaves invariant both $G_{1}$ and a maximal compact subgroup $K_{1} \subset G_{1}$. Then there exists a maximal compact subgroup $K_{2} \subset G_{2}$, containing $K_{1}$ and invariant under each automorphism from $\Delta$.

Proof Consider the semidirect product $\tilde{G}_{2}=G_{2} \rtimes \Delta$, where $G_{2}$ is normal in $\tilde{G}_{2}$, with the given action of $\Delta$ on $G_{2}$ as an automorphism group. We identify $\Delta$ with the corresponding subgroup in $\tilde{G}_{2}$. The semidirect products $\tilde{G}_{1}:=G_{1} \rtimes \Delta$ and $\tilde{K}_{1}:=K_{1} \rtimes \Delta$ are subgroups of $\tilde{G}_{2}$.

Maximal compacts subgroups of a Lie group with finitely many connected components are characterized by the two properties (see [Mo]): (i) such a subgroup has non-empty intersection with every connected component of the ambient group; (ii) its intersection with the connected component of the identity is a maximal compact subgroup in that component. It follows from this criterion that $\tilde{K}_{1}$ is a maximal compact subgroup of $\tilde{G}_{1}$. Let $\tilde{K}_{2}$ be a maximal compact subgroup of $\tilde{G}_{2}$ containing $\tilde{K}_{1}$. Since $\tilde{K}_{2}$ has non-empty intersection with all connected components of $\tilde{G}_{2}$ including those which are contained in $G_{2}$ (and are therefore connected components of $G_{2}$ ) and, also, $\left(\tilde{G}_{2}\right)^{\circ}=G_{2}^{\circ}$, we see from the above criterion that $K_{2}:=\tilde{K}_{2} \cap G_{2}$ is a maximal compact subgroup of $G_{2}$. Because $K_{1} \subset \tilde{K}_{1} \subset \tilde{K}_{2}$, we have $K_{1} \subset K_{2}$. Furthermore, $\Delta \subset \tilde{K}_{1} \subset \tilde{K}_{2}$. Thus, if $k \in K_{2}, \delta \in \Delta$ then $\delta k \delta^{-1} \in \tilde{K}_{2}$. Since $\delta G_{2} \delta^{-1}=G_{2}$, we also have $\delta k \delta^{-1} \in G_{2}$, showing that $K_{2}$ is $\delta$-invariant.

\section{Antilinear $\theta$-equivariant involutions of $K$-modules}

For a complex vector space $V$ let $\bar{V}$ denote the conjugate vector space. By definition, $\bar{V}$ coincides with $V$ as a real vector space, whereas the multiplication by complex numbers on $\bar{V}$ is given by $(c, v) \mapsto \bar{c} v \quad(c \in \mathbb{C}, v \in V)$. Let $\iota: V \rightarrow \bar{V}$ denote the identity map of real vector spaces, which is antilinear with respect to the complex structures on $V$ and $\bar{V}$. If $\varrho: K \rightarrow \mathrm{GL}(V)$ is a representation of a group $K$ then there is a natural representation of $K$ in $\bar{V}$ which we denote by $\bar{\varrho}$. We have $\iota(\varrho(k) v)=\bar{\varrho}(k) \iota(v)$ for all $k \in K, v \in V$.

Theorem 3.1 Let $K$ be a connected compact Lie group, $\theta: K \rightarrow K$ a Weyl involution, and $\varrho: K \rightarrow \mathrm{GL}(V)$ a complex representation of $K$. Then there exists an antilinear mapping $\mu: V \rightarrow V$, such that $\mu^{2}=$ Id and

$$
\mu(\varrho(k) v)=\varrho(\theta(k)) \mu(v)
$$


for all $k \in K, v \in V$. If $\varrho$ is irreducible and $\mu^{\prime}: V \rightarrow V$ is another mapping with the above properties then $\mu^{\prime}=c \mu$ for some $c \in \mathbb{C},|c|=1$.

Proof A $K$-invariant positive Hermitian form on $V$ gives rise to an isomorphism of $K$ modules $\bar{V} \simeq V^{*}$. Let $V^{\theta}$ be the $K$-module defined by the representation $k \mapsto \varrho(\theta(k))$ in $V$. Then, by the main property of a Weyl involution, the $K$-modules $V^{\theta}$ and $\bar{V}$ are also isomorphic. Thus we have an isomorphism of complex vector spaces $A: V \rightarrow \bar{V}$ such that

$$
A(\varrho(k) v)=\bar{\varrho}(\theta(k)) A v
$$

for all $k \in K, v \in V$. Define the map $\mu: V \rightarrow V$ by $\mu=\iota^{-1} \cdot A$. Then $\mu$ is antilinear and $\mu(\varrho(k) v)=\varrho(\theta(k)) \mu(v)$.

Note that it is enough to prove the theorem for irreducible representations. Indeed, if $V$ is the direct sum of irreducible $K$-modules $V_{j}$ and $\mu_{j}: V_{j} \rightarrow V_{j}$ has the required property for each $j$ then $\mu$ can be defined as the direct sum of $\mu_{j}$. We assume now that $V$ is an irreducible $K$-module. Let $T \subset K$ be a maximal torus on which $\theta$ acts as the inversion and let $v \in V$ be a weight vector, such that the corresponding weight $\lambda: T \rightarrow \mathbb{C}^{*}$ has multiplicity one. For the map $\mu$ constructed above and for any $t \in T$ we have

$$
\varrho(t)^{-1} \mu(v)=\varrho(\theta(t)) \mu(v)=\mu(\varrho(t) v)=\mu(\lambda(t) v)=\overline{\lambda(t)} \mu(v)
$$

or, equivalently,

$$
\varrho(t) \mu(v)=\lambda(t) \mu(v) .
$$

Therefore $\mu(v)=a v$, where $a \in \mathbb{C}^{*}$. Applying $\mu$ again we get $\mu^{2}(v)=|a|^{2} v$. Since $V$ is an irreducible $K$-module and $\mu^{2}$ is a (complex linear) intertwining operator, it follows by Schur's Lemma that $\mu^{2}=|a|^{2} \cdot$ Id. Replacing $\mu$ by $|a|^{-1} \mu$ we obtain the mapping with all required properties. Finally, assume $\mu^{\prime}$ and $\mu$ are two such mappings. Then $\mu(v)=a v$ and $\mu^{\prime}(v)=a^{\prime} v$ as above. Because both $\mu^{2}$ and $\mu^{\prime 2}$ are the identity maps, it follows that $|a|=\left|a^{\prime}\right|=1$. Since $\mu \mu^{\prime}$ is a (complex linear) intertwining operator and $\mu \mu^{\prime}(v)=a\left(a^{\prime}\right)^{-1} v$, we have $\mu \mu^{\prime}=a\left(a^{\prime}\right)^{-1} \cdot$ Id. Multiplying this equality by $\mu$ on the left, we get $\mu^{\prime}=c \mu$, where $c=a^{\prime} a^{-1},|c|=1$.

\section{Involution $\sigma$ and spherical subgroups}

Let $H \subset G$ be an algebraic subgroup, $\phi \in \mathrm{X}(H)$, and $\mathbb{L}^{\phi}$ the homogeneous line bundle over $G / H$ associated with the character $\phi$. Recall that $\mathbb{L}^{\phi}=G \times_{H} \mathbb{C}$, where the action of $H$ on $G \times \mathbb{C}$ is given by

$$
(g, z) \stackrel{h}{\mapsto}\left(g h^{-1}, \phi(h) z\right) \quad(g \in G, h \in H, z \in \mathbb{C}) .
$$

The space of regular sections $\Gamma\left(G / H, \mathbb{L}^{\phi}\right)$ is a $G$-module. Namely, the elements of $\Gamma\left(G / H, \mathbb{L}^{\phi}\right)$ are identified with the regular functions on $G$ satisfying the equation $f(g h)=\phi(h)^{-1} f(g)$, where $g \in G, h \in H$. Under this identification, $G$ acts in 
$\Gamma\left(G / H, \mathbb{L}^{\phi}\right)$ by left translations. We write $(g \cdot f)(x)=f\left(g^{-1} x\right)$ for $g, x \in G$ and $f \in \Gamma\left(G / H, \mathbb{L}^{\phi}\right)$.

Let $N=N_{G}(H)$ and assume that

$$
\phi\left(u h u^{-1}\right)=\phi(h) \quad \text { for } h \in H, u \in N .
$$

Since $\mathrm{X}(H)$ is a discrete group, this is automatic for $u \in H N^{\circ}$. Thus the orbit of $\phi$ under $N$ acting by conjugation is finite. This will be used in the proof of Theorem 4.3. It follows from (2) that $\operatorname{Ker} \phi$ is normal in $N$. Also, $\Gamma\left(G / H, \mathbb{L}^{\phi}\right)$ has a structure of an $\mathrm{N}$-module with the action given by

$$
f \mapsto f^{u}, f^{u}(g)=f(g u), u \in N .
$$

Indeed, if $f$ is a section of $\mathbb{L}^{\phi}$ then

$$
f^{u}(g h)=f(g h u)=f\left(g u \cdot u^{-1} h u\right)=\phi\left(u^{-1} h u\right)^{-1} f(g u)=\phi(h)^{-1} f^{u}(g),
$$

where the last equality follows from (2). This shows that $f^{u}$ is also a section of $\mathbb{L}^{\phi}$. We refer to this action as the right action of $N$ in $\Gamma\left(G / H, \mathbb{L}^{\phi}\right)$.

Recall that an algebraic subgroup $H \subset G$ is called spherical if the homogeneous space $G / H$ is a spherical variety. In what follows, we are mainly interested in spherical subgroups $H \subset G$. Though this fact is not used in the proofs below, it is good to remember that for $H$ spherical the group $N / H$ is diagonalizable and, in particular, abelian; see [BP].

Theorem 4.1 Let $H \subset G$ be a spherical subgroup, $a \in G$, and $\sigma(H)=a H a^{-1}$. Let $\phi \in \mathrm{X}(H)$ be a character satisfying (2). Assume in addition that

$$
\phi\left(a^{-1} \sigma(h) a\right)=\overline{\phi(h)}
$$

for all $h \in H$. Then any irreducible $G$-submodule $V \subset \Gamma\left(G / H, \mathbb{L}^{\phi}\right)$ is invariant under the right action of $N$. Moreover, $N$ acts on $V$ by

$$
f^{u}=\phi_{V}(u)^{-1} \cdot f \quad(f \in V, u \in N),
$$

where the character $\phi_{V} \in \mathrm{X}(N)$ satisfies

$$
\overline{\phi_{V}(u)}=\phi_{V}\left(\sigma\left(a u a^{-1}\right)\right)
$$

(this makes sense because $\left.\sigma(N)=a N a^{-1}\right)$.

Proof Fix a section $f \in \Gamma\left(G / H, \mathbb{L}^{\phi}\right)$ and put

$$
F(g)=\overline{f(\sigma(g) a)}
$$


Clearly, $F$ is a regular function on $G$, and

$$
\begin{gathered}
F(g h)=\overline{f(\sigma(g) \sigma(h) a)}=\overline{f\left(\sigma(g) a \cdot a^{-1} \sigma(h) a\right)}= \\
\quad=\overline{\phi\left(a^{-1} \sigma(h) a\right)^{-1}} \cdot \overline{f(\sigma(g) a)}=\phi(h)^{-1} F(g)
\end{gathered}
$$

in view of (3). Therefore $F \in \Gamma\left(G / H, \mathbb{L}^{\phi}\right)$, and so we obtain the antilinear map

$$
\eta: \Gamma\left(G / H, \mathbb{L}^{\phi}\right) \rightarrow \Gamma\left(G / H, \mathbb{L}^{\phi}\right), \quad \eta(f)(g)=\overline{f(\sigma(g) a)} .
$$

For $k \in K$ we have

$$
\begin{gathered}
\eta(k \cdot f)(g)=\overline{(k \cdot f)(\sigma(g) a)}=\overline{f\left(k^{-1} \sigma(g) a\right)}=\overline{f\left(\sigma\left(\theta(k)^{-1} g\right) a\right)}= \\
=\eta(f)\left(\theta(k)^{-1} g\right)=[\theta(k) \cdot \eta(f)](g)
\end{gathered}
$$

because $\theta$ and $\sigma$ coincide on $K$. Therefore $\eta(V)$ is a $K$-submodule and hence a $G$ submodule in $\Gamma\left(G / H, \mathbb{L}^{\phi}\right)$. Moreover, the mapping $\eta: V \rightarrow \eta(V)$ gives rise to a $K$ equivariant antilinear bijective map $V \rightarrow \eta(V)^{\theta}$, where the $K$-module $\eta(V)^{\theta}$ is obtained from $\eta(V)$ by changing the corresponding representation $k \mapsto \varrho(k)$ to $k \mapsto \varrho(\theta(k))(k \in$ $K)$. Let $\bar{V}$ be the complex conjugate space of $V$ with the natural representation of $K$. As we have seen, there is an isomorphism of $K$-modules $\bar{V} \simeq \eta(V)^{\theta}$. On the other hand, from the existence of a $K$-invariant positive Hermitian form on $V$ we get an isomorphism of $K$-modules $\bar{V} \simeq V^{*}$. Furthermore, since $\theta$ is a Weyl involution, we also have the isomorphism $\eta(V)^{\theta} \simeq \eta(V)^{*}$. Combining these isomorphisms, we conclude that $V^{*} \simeq \eta(V)^{*}$ and $V \simeq \eta(V)$. But $H$ is a spherical subgroup in $G$, and so each irreducible $G$-submodule in $\Gamma\left(G / H, \mathbb{L}^{\phi}\right)$ has multiplicity one. Thus $\eta(V)=V$.

The action of $G$ commutes with the right action of $N$ on $\Gamma\left(G / H, \mathbb{L}^{\phi}\right)$. Since $\Gamma\left(G / H, \mathbb{L}^{\phi}\right)$ is multiplicity free, $V$ is invariant under the action of $N$. By Schur's Lemma $N$ acts on $V$ via multiplication by a character. We denote the latter one by $\phi_{V}(u)^{-1}, u \in N$. In other words, for $f \in V$ we have

$$
f(g u)=\phi_{V}(u)^{-1} f(g) .
$$

Replacing $g$ by $\sigma(g) a$, we get

$$
f(\sigma(g) a u)=\phi_{V}(u)^{-1} f(\sigma(g) a)=\phi_{V}(u)^{-1} \cdot \overline{\eta(f)(g)} .
$$

On the other hand, let $u^{\prime}=\sigma\left(a u a^{-1}\right)$. Then

$$
f(\sigma(g) a u)=f\left(\sigma(g) a u a^{-1} a\right)=f\left(\sigma\left(g u^{\prime}\right) a\right)=\overline{\eta(f)\left(g u^{\prime}\right)}=\overline{\phi_{V}\left(u^{\prime}\right)^{-1}} \cdot \overline{\eta(f)(g)},
$$

where in the last equality we used that $\eta(f) \in V$. Together with the previous computation, this proves (4).

Corollary 4.2 Let $H \subset G$ be a spherical subgroup, such that $\sigma(H)=a H a^{-1}$ for some $a \in G$. Assume that $\phi \in \mathrm{X}(H)$ satisfies (2) and (3) and let $U \subset N$ be a Lie subgroup 
with compact image in $N / \operatorname{Ker} \phi$. Then for any $u \in U$ the right action of $\sigma\left(a u a^{-1}\right)$ on $\Gamma\left(G / H, \mathbb{L}^{\phi}\right)$ coincides with that of $u^{-1}$.

Proof Applying Lemma 2.1 to the epimorphism $N \rightarrow N / \operatorname{Ker} \phi$, we can write any $u \in U$ as $u=c h$, where $h \in H, \phi(h)=1$, and $c$ is contained in a compact subgroup of $N$. Let $V \subset \mathbb{C}[G / H]$ be an irreducible $G$-submodule. Since $\left.\phi_{V}\right|_{H}=\phi$ by the definition of $\phi_{V}$, we have $\phi_{V}(u)=\phi_{V}(c)$ and, therefore, $\left|\phi_{V}(u)\right|=1$. By Theorem 4.1, it follows from (4) that

$$
\phi_{V}\left(u^{-1}\right)=\phi_{V}\left(\sigma\left(a u a^{-1}\right)\right)
$$

This is true for any $G$-submodule $V \subset \mathbb{C}[G / H]$, showing that $u^{-1}$ and $\sigma\left(a u a^{-1}\right)$ induce the same linear transform of $\Gamma\left(G / H, \mathbb{L}^{\phi}\right)$.

Theorem 4.3 Let $H \subset G$ be a spherical subgroup, $a \in G$, and $\sigma(H)=a H a^{-1}$. Let $U \subset N$ be a Lie subgroup with compact image in $N / H$. Then

$$
\sigma\left(a u a^{-1}\right) \equiv u^{-1} \quad(\bmod H)
$$

for all $u \in U$. In particular, if $H \subset U$ then $U$ is stable under the mapping $u \mapsto \sigma\left(a_{a a^{-1}}\right)$ and the induced automorphism of $U / H$ is the inversion.

Proof By Chevalley's theorem there exist a rational representation $\rho: G \rightarrow \mathrm{GL}(V)$ and a vector $v \in V$, such that

$$
H=\{g \in G \mid \rho(g) v \in \mathbb{C} \cdot v\}
$$

Let $\phi \in \mathrm{X}(H)$ denote the corresponding character, i.e., $\rho(h) v=\phi(h) \cdot v$ for $h \in H$. For $u \in N$ define $\phi^{u} \in \mathrm{X}(H)$ by

$$
\phi^{u}(h)=\phi\left(u h u^{-1}\right) \quad(h \in H) .
$$

Since the family $\left\{\phi^{u}\right\}_{u \in N}$ is finite, we can find $u_{1}, u_{2}, \ldots, u_{k} \in N$ with the property that for every $u \in N$ there exists exactly one $u_{i}, i \in[1, k]$, such that $\phi^{u}=\phi^{u_{i}}$. Write $\phi_{i}$ instead of $\phi^{u_{i}}$, define the vectors $v_{i} \in V$ by $v_{i}=\rho\left(u_{i}\right) v$, and consider the tensor product of representations $\tilde{\rho}: G \rightarrow \operatorname{GL}(\tilde{V})$, where $\tilde{V}=V \otimes \ldots \otimes V$ (k times). For the decomposable vector $\tilde{v}=v_{1} \otimes \ldots \otimes v_{k} \in \tilde{V}$ we have

$$
H=\{g \in G \mid \tilde{\rho}(g) \tilde{v} \in \mathbb{C} \cdot \tilde{v}\}
$$

and the corresponding character of $H$ is the product

$$
\tilde{\phi}=\prod_{i=1}^{k} \phi_{i}
$$

which is invariant under $N$, i.e., $\tilde{\phi}\left(u h u^{-1}\right)=\tilde{\phi}(h)$ for all $h \in H, u \in N$. Therefore, replacing the representation $\rho: G \rightarrow \mathrm{GL}(V)$ and the vector $v \in V$ by $\tilde{\rho}: G \rightarrow \tilde{G L}(\tilde{V})$ and, respectively, $\tilde{v} \in \tilde{V}$ we may assume that $\phi$ satisfies (2). 
We now want to change the triple $V, v$ and $\rho$ without changing $H$, so that $\phi$ also satisfies (3). In the notation of Sect. 3, let $\bar{V}$ denote the complex conjugate vector space. We have the antilinear map $\iota: V \rightarrow \bar{V}$ and the antiholomorphic representation $\bar{\rho}: G \rightarrow \operatorname{GL}(\bar{V})$ such that $\iota(\rho(g))=\bar{\rho}(g) \iota$ for $g \in G$. Define a representation of $G$ in the tensor product $V \otimes \bar{V}$ as $g \mapsto \rho(g)$ on the first factor and $g \mapsto \bar{\rho}\left(a^{-1} \sigma(g) a\right)$ on the second one. Note that this representation is rational. Moreover, it is easily seen that $H$ is the stabilizer of the line $\mathbb{C} \cdot v \otimes \iota(v)$ and the corresponding character is given by

$$
\hat{\phi}(h)=\phi(h) \cdot \overline{\phi\left(a^{-1} \sigma(h) a\right)}, \quad h \in H .
$$

Since $\sigma(a) a \in N$ and $\phi^{u}=\phi$ for $u \in N$, we see that $\hat{\phi}$ satisfies (3).

We just proved that there exists an equivariant embedding $j: G / H \rightarrow \mathbb{P}(V)$ with $j^{*} \mathcal{O}(-1)=\mathbb{L}^{\phi}$. Thus the sections of the dual bundle $\left(\mathbb{L}^{\phi}\right)^{*}=\mathbb{L}^{\varphi}$, where $\varphi=\phi^{-1}$, separate points of $G / H$. It follows that the ineffectivity kernel of the right action of $N$ in $\Gamma\left(G / H, \mathbb{L}^{\varphi}\right)$ is exactly $H$. If $U \subset N$ is a Lie subgroup with compact image in $N / H$, then by Lemma 2.1 there exists a possibly larger Lie subgroup of $N$ with compact image in $N / \operatorname{Ker} \varphi$, which is mapped onto $U H / H$. We denote this larger subgroup again by $U$. Let $u \in U$. Then, by Corollary 4.2, $u^{-1}$ and $\sigma\left(a u a^{-1}\right)$ induce the same transform of $\Gamma\left(G / H, \mathbb{L}^{\varphi}\right)$. Therefore $\sigma\left(a u a^{-1}\right) \equiv u^{-1}$ modulo $H$.

As a corollary, we get another proof of Proposition 5.2 in [AV].

Corollary 4.4 (see [AV], Proposition 5.2) Let $H \subset G$ be a reductive spherical subgroup invariant under a Weyl involution $\theta$. Then $\theta$ acts as the inversion on the abelian group $N / H$.

Proof Take $\Delta=\{e, \theta\}$ in Lemma 2.2 and apply the assertion of the lemma to each of the three inclusions $\{e\} \subset H \subset N \subset G$, moving from the left to the right. Then we get $\theta$-stable maximal compact subgroups in $H, N$ and $G$ together with similar inclusions. In other words, there is a $\theta$-stable maximal compact subgroup $K \subset G$, such that $K \cap H$ and $K \cap N$ are maximal compact subgroups in $H$ and, respectively, in $N$. Let $\tau$ be the Cartan involution of $G$ with $G^{\tau}=K$. Clearly, $\theta \tau=\tau \theta$. Since $\tau$ fixes pointwise the maximal compact subgroups $K \cap H$ and $K \cap N$ of the reductive groups $H$ and $N$, it follows that $\tau(H)=H$ and $\tau(N)=N$. Thus $\tau$ acts on $N / H$. Note that $N / H$ is a reductive algebraic group and $(K \cap N) /(K \cap H)$ is its maximal compact subgroup. By Theorem 4.3 the product $\sigma=\tau \theta$ and therefore also $\theta$ acts as the inversion on $(K \cap N) /(K \cap H)$, showing that the latter group is abelian. (Of course, it follows that $N / H$ is abelian, but this fact is known in a more general setting; see $[\mathrm{BP}])$. Since $\theta$ is an algebraic automorphism, $\theta$ acts as the inversion on the whole group $N / H$.

\section{Proof of main results}

Proof of Theorem 1.2 First of all, we reduce the statement to the algebraic case. Namely, by Theorem 2 in $[\mathrm{AH}], X$ can be embedded as a $K$-invariant domain into an affine 
spherical $K^{\mathbb{C}}$-variety which we denote by $X^{\mathbb{C}}$. Moreover, if $G=K^{\mathbb{C}}$ and $i: X \hookrightarrow X^{\mathbb{C}}$ is the embedding then $G \cdot i(X)=X^{\mathbb{C}}$. Hence $X^{\mathbb{C}}$ is non-singular. Assuming that the theorem is proved for $X^{\mathbb{C}}$, we get a $\theta$-equivariant antiholomorphic involution $\mu: X^{\mathbb{C}} \rightarrow$ $X^{\mathbb{C}}$. Since the variety $X^{\mathbb{C}}$ is spherical, its Fréchet algebra $\mathcal{O}(X)$ is a multiplicity free $K$-module. Thus, by Theorem 4.1 in $[\mathrm{AP}]$, we have $\mu(x) \in K x$ for all $x \in X^{\mathbb{C}}$. It follows that $\mu(i(X))=i(X)$, and so the required involution of $X$ is obtained from $\mu$ by restriction.

From now on we assume that $X$ is a smooth affine spherical variety of $G$. Since $\mathbb{C}[X]=\mathbb{C}$, the well-known application of Luna's Slice Theorem displays $X$ as a vector bundle. Namely, $X=G \times{ }_{H} V$, where $H$ is a reductive subgroup of $G$ and $V$ is an $H$-module; see [Lu], Cor.2, p.98. Moreover, since $X$ is a spherical variety, it follows that $H$ is a spherical subgroup in $G$ and $V$ is a spherical $H$-module; see [K-VS], Corollary 2.2 .

It suffices to prove our theorem for some maximal compact subgroup $K \subset G$ and for some Weyl involution $\theta$ of $G$ satisfying $\theta(K)=K$. Let $L$ be a maximal compact subgroup of $H$ contained in $K$. According to [AV], Prop. 5.14, we can find a Weyl involution $\theta$ of $G$ so that $K$ and $L$ are $\theta$-invariant and $\theta$ induces a Weyl involution of $K$ and $L^{\circ}$. Then, of course, $\theta(H)=H$ and $\left.\theta\right|_{H^{\circ}}$ is a Weyl involution of $H^{\circ}$. Moreover, if $\tau: G \rightarrow G$ is the Cartan involution with $G^{\tau}=K$ then $\tau \theta=\theta \tau$. We will use our standard notation $\sigma=\tau \theta$.

By Theorem 3.1 there exists an antilinear involution $\nu: V \rightarrow V$, such that $\nu(l v)=$ $\theta(l) \nu(v)$ for $v \in V$ and $l \in L^{\circ}$. Since $L^{\circ}$ is a maximal compact subgroup of the connected reductive group $H^{\circ}$, it follows that $\nu(h v)=\sigma(h) \nu(v)$ for $h \in H^{\circ}, v \in V$. Indeed, if $v$ is fixed then this equality holds for all $h \in L^{\circ}$. But $L^{\circ}$ is a maximal totally real submanifold in $H^{\circ}$, and so the above equality holds also on $H^{\circ}$. We claim that in fact

$$
\nu(h v)=\sigma(h) \nu(v) \quad \text { for all } v \in V, h \in H .
$$

Proof of $(*)$. Take a vector $v_{0} \in V$ such that the orbit $G v_{0}$ is open in $X$. Then the orbit $H v_{0}$ is open in $V$. Let $U=G_{v_{0}}=H_{v_{0}}$ be the isotropy subgroup at $v_{0}$. Put $v_{1}=\nu\left(v_{0}\right)$. Then $\nu\left(h v_{0}\right)=\sigma(h) v_{1}$ for $h \in H^{\circ}$, hence $\nu\left(H^{\circ} v_{0}\right)=H^{\circ} v_{1}$. But the open orbit $H v_{0} \subset V$ is connected. Thus $H^{\circ}$ acts on this orbit transitively, i.e., $H v_{0}=H^{\circ} v_{0}$. Since the orbit $H^{\circ} v_{1}$ is also open, we have $H^{\circ} v_{0}=H^{\circ} v_{1}$ by the uniqueness of an open orbit. Therefore $v_{1}=a v_{0}$, where $a \in H^{\circ}$.

Since $U \subset H$, the intersection $U \cap H^{\circ}$ has finite index in $U$. Thus $G /\left(U \cap H^{\circ}\right)$ is a spherical variety together with $G / U$. The equality $v_{1}=a v_{0}$ shows that the isotropy subgroup of $v_{1}$ in $H^{\circ}$ is $a\left(U \cap H^{\circ}\right) a^{-1}$. On the other hand, $\nu$ is $\sigma$-equivariant with respect to the connected group $H^{\circ}$. From this it follows that $a\left(U \cap H^{\circ}\right) a^{-1}=\sigma\left(U \cap H^{\circ}\right)$. We now apply Theorem 4.3 to the pair $U \cap H^{\circ} \subset G$ and to the subgroup $U$ normalizing $U \cap H^{\circ}$. The quotient $U /\left(U \cap H^{\circ}\right)$ is finite, and so we obtain $\sigma(U)=a U a^{-1}$.

Since $H^{\circ}$ is transitive on $H v_{0}$, we have $H=H^{\circ} \cdot U$. For any $h \in H$ write $h=h^{\prime} u$ with $h^{\prime} \in H^{\circ}, u \in U$. Then

$$
\nu\left(h v_{0}\right)=\nu\left(h^{\prime} u v_{0}\right)=\nu\left(h^{\prime} v_{0}\right)=\sigma\left(h^{\prime}\right) v_{1}
$$


and, on the other hand,

$$
\sigma(h) v_{1}=\sigma\left(h^{\prime}\right) \sigma(u) v_{1}=\sigma\left(h^{\prime}\right) a u^{\prime} a^{-1} v_{1}=\sigma\left(h^{\prime}\right) v_{1}
$$

where $\sigma(u)=a u^{\prime} a^{-1}$ and $u^{\prime} \in U$. These computations show that $\nu\left(h v_{0}\right)=\sigma(h) v_{1}$ for any $h \in H$. Replacing $h$ by $h h^{\prime}$ where $h^{\prime} \in H^{\circ}$, we get $\nu\left(h h^{\prime} v_{0}\right)=\sigma\left(h h^{\prime}\right) v_{1}=$ $\sigma(h) \sigma\left(h^{\prime}\right) v_{1}=\sigma(h) \nu\left(h^{\prime} v_{0}\right)$. Hence $\nu(h v)=\sigma(h) \nu(v)$ where $v=h^{\prime} v_{0}$ is any vector from the open orbit in $V$. Since the open orbit is dense, this completes the proof of $(*)$.

Let $\mu$ be the self-map of $G \times V$ defined by

$$
\mu(g, v)=(\sigma(g), \nu(v))
$$

Obviously, $\mu$ is an antiholomorphic involution of $G \times V$. For $h \in H$ let $t_{h}(g, v)=$ $\left(g h^{-1}, h v\right)$. This defines an action of $H$ on $G \times V$ and, by definition, $X$ is the geometric quotient with respect to this action. It follows from $(*)$ that $\mu \cdot t_{h}=t_{\sigma(h)} \cdot \mu$. Thus $\mu$ defines a self-map of $X$ with all required properties.

Proof of Theorem 1.1 As we already noted in the introduction, the existence of an antiholomorphic involution $\mu: X \rightarrow X$ with property (1) implies that $X$ is spherical; see [FT], Theorem 3. Conversely, if $X$ is a spherical Stein manifold then, by Theorem 1.2, there exists a $\theta$-equivariant antiholomorphic involution $\mu: X \rightarrow X$. On the other hand, a holomorphically separable complex $K$-manifold with a $\theta$-equivariant antiholomorphic involution is multiplicity free if and only if (1) is fulfilled; see [AP], Theorem 4.1.

\section{References}

[AV] D.N.Akhiezer, E.B.Vinberg, Weakly symmetric spaces and spherical varieties, Transformation Groups 4 (1999), 3 - 24

[AH] D.Akhiezer, P.Heinzner, Spherical Stein spaces, Manuscripta Math. 114 (2004), $327-334$

[AP] D.Akhiezer, A.Püttmann, Antiholomorphic involutions of spherical complex spaces, Proc.Amer.Math.Soc., posted on January 3, 2008, PII S 0002-9939(08)08988-0 (to appear in print)

[BP] M.Brion, F.Pauer, Valuations des espaces homogènes sphériques, Comment. Math. Helv. 62 (1987), 265 - 285

[FT] J.Faraut, E.G.F.Thomas, Invariant Hilbert spaces of holomorphic functions, J. of Lie Theory, 9 (1999), 383 - 402

[He] S.Helgason, Differential Geometry, Lie Groups, and Symmetric Spaces, Acad. Press, 1978. 
[K-VS] F.Knop, B. Van Steirteghem, Classification of smooth affine spherical varieties, Transformation Groups, 11 (2006), 495 - 516

[L] D.Luna, Slices étales, Mem.Soc.Math.France (N.S.) 33 (1973), 81 - 105

[M] D.Montgomery, Simply connected homogeneous spaces, Proc.Amer.Math.Soc. 1 (1950), $467-469$

[Mo] G.D.Mostow, Self-adjoint groups, Annals of Math. 62 (1955), 44 - 55

[Se] F.J.Servedio, Prehomogeneous vector spaces and varieties, Trans.Amer.Math.Soc. 176 (1973), 421 - 444

[VK] E.B.Vinberg, B.N.Kimel'feld, Homogeneous domains in flag manifolds and spherical subgroups of semisimple Lie groups, Funktsional'nyi Analiz i ego Prilozheniya 12 (1978), 12 - 19 (Russian); English translation: Funct.Anal.Appl. 12 (1978), 168 - 174

Institute for Information Transmission Problems, B.Karetny per. 19, Moscow, 101447, Russia 